\title{
THE UNIQUENESS OF BOUNDED OR MEASURABLE SOLUTIONS OF SOME FUNGTIONAL EQUATIONS
}

T. D. HOWROYD

(Received 13 March 1968; revised 18 November 1968)

In this paper we are concerned with the uniqueness of solutions of functional equations of the form

$$
\begin{aligned}
& g(F(x, y))=H(f(x), f(y) ; x, y), \\
& f(F(x, y))=H(f(x), f(y) ; x, y) .
\end{aligned}
$$

Some conditions for (1) or (2) to have at most one real continuous solution $f$ which satisfies two given initial conditions are contained in [2], [3], [4] and $[7]$. Conditions sufficient for the equation

$$
f(x+y)=G(f(x), f(y))
$$

to determine at most one continuous solution $f$ with values in a Banach algebra are contained in [5]. It is well known (see [1] ch. 2) that one initial condition suffices for Cauchy's equation

$$
f(x+y)=f(x)+f(y)
$$

or two for Jensen's equation

$$
f\left(\frac{1}{2} x+\frac{1}{2} y\right)=\cdot \frac{1}{2} f(x)+\frac{1}{2} f(y)
$$

to uniquely determine a real solution $f$ which is bounded on an interval or majorized on a set of positive measure by a measurable function. We place conditions on $F$ and $H$ so that similar statements can be made about solutions of (1) or (2). The corresponding results for solutions which are functions of many real variables follow as for Cauchy's and Jensen's equations (see [1] ch. 5).

In the following $(\Delta, d)$ is a metric space; $D \subset \Delta ; C$ is the complex plane; $R$ is the real line; $R^{n}$ is $n$-dimensional Euclidean space; $I$ is a real interval or a convex set in $R^{n}$. If $f: I \rightarrow \Delta$ is bounded in a neighbourhood of each point of $I$ we say $f$ is locally bounded in $I$. If $D=R$ or $C$ we take $d(u, v)=|u-v|, u, v \in D$. We use a square (round) bracket to denote a closed (open) end of an interval.

THEOREM 1. Let $I$ be an interval. Let $F: I \times I \rightarrow R$ be continuous and 186 
strictly increasing (or decreasing). Let $H: D \times D \times I \times I \rightarrow \Delta$ be such that

$$
H(u, v ; x, y)=H(U, v ; x, y) \text { iff } u=U,
$$

or

$$
H(u, v ; x, y)=H(u, V ; x, y) \text { iff } v=V ;
$$

$\left(\mathrm{H}_{2}\right)$ corresponding to every bounded set $J \subset I$ there exists $r \in\left[\frac{1}{2}, 1\right)$ and $s>0$ such that in $D \times D \times J \times J$

$$
\begin{aligned}
& d\{H(u, v ; x, y), H(U, V ; x, y)\} \leqq r s\{d(u, U)+d(v, V)\}, \\
& d\{H(u, u ; x, x), H(v, v ; x, x)\} \geqq s d(u, v) .
\end{aligned}
$$

Let $a, b \in I$ and $a<b$. Then corresponding to any given $f(a)$ and $f(b)$ there is at most one pair of functions $f: I \rightarrow D$ and $g:\{F(x, y): x, y \in I\} \rightarrow \Delta$ such that $f$ is locally bounded in $I$ and (1) holds.

Proof. Let $z=G(x, y)$ be the unique solution of $F(z, z)=F(x, y)$. If $F(x, x) \equiv x\left(F\right.$ is reflexive), then $G=F$. Let $\alpha_{1}(x)=G(a, x)$ and $\alpha_{2}(x)=G(x, b), x \in I$; then $\alpha_{1}$ and $\alpha_{2}$ are continuous, strictly increasing, $\alpha_{1}(a)=a, \alpha_{1}(b)=\alpha_{2}(a), \alpha_{2}(b)=b$. Hence $\alpha_{1}$ and $\alpha_{2}$ together map $[a, b]$ onto $[a, b]$.

Let $H(u, u ; x, x)=h(u ; x)$. If (1) holds in $I$ the substitution of $G(x, y)$ for both $x$ and $y$ shows that

$$
h\{f(G(x, y)) ; G(x, y)\}=H(f(x), f(y) ; x, y), x, y \in I .
$$

If $f(a)=A$ and $f(b)=B$ then

$$
\begin{aligned}
& h\left\{f\left(\alpha_{1}(x)\right) ; \alpha_{1}(x)\right\}=H(A, f(x) ; a, x), x \in I, \\
& h\left\{f\left(\alpha_{2}(x)\right) ; \alpha_{2}(x)\right\}=H(f(x), B ; x, b), x \in I .
\end{aligned}
$$

Let $f_{1}$ and $f_{2}$ satisfy (5) and (6) and be locally bounded in $I$. If $x_{0} \in[a, b]$ and $n$ is a positive integer there exist $p_{1}, \cdots, p_{n} \in\{1,2\}$ and $x_{1}, \cdots, x_{n} \in[a, b]$ such that $x_{0}=\alpha_{p_{1}}\left(x_{1}\right), x_{k}=\alpha_{p_{k+1}}\left(x_{k+1}\right), k=1, \cdots, n-1$, and hence (5), (6) and $\left(\mathrm{H}_{2}\right)$ imply that there exist $r \in\left[\frac{1}{2}, 1\right)$ and $s>0$ such that

$$
\begin{aligned}
s d\left\{f_{1}\left(x_{0}\right), f_{2}\left(x_{0}\right)\right\} & \leqq d\left\{h\left(f_{1}\left(x_{0}\right) ; x_{0}\right), h\left(f_{2}\left(x_{0}\right) ; x_{0}\right)\right\} \\
& \leqq r s d\left\{f_{1}\left(x_{1}\right), f_{2}\left(x_{1}\right)\right\} \\
& \leqq r^{n} s d\left\{f_{1}\left(x_{n}\right), f_{2}\left(x_{n}\right)\right\}
\end{aligned}
$$

which decreases to 0 as $n \rightarrow \infty$ since $f_{1}$ and $f_{2}$ are bounded on $[a, b]$. Hence $f_{1}=f_{2}$ in $[a, b]$. As in [7] the condition $\left(\mathrm{H}_{1}\right)$ ensures that $f_{1}=f_{2}$ in $I$.

Remarks. If $I=[a, b]$ the condition $\left(\mathrm{H}_{1}\right)$ is unnecessary; and if also $F$ is reflexive then $F$ need only be non-decreasing (or non-increasing). On the other hand if (1) is replaced by (2) and $a \neq F(a, a) \in I$ then the 
condition $f(b)=B$ is redundant (cf. [4], [6]) - this remark also applies to the next theorem. If $H(u, u ; x, x) \equiv u$ then (3) and (4) are equivalent to (3) with $s=1$.

Theorem 2. Let I be an interval. Let $F$ satisfy the hypotheses of Theorem 1 and corresponding to each bounded set $J \subset I$ let there exist positive constants $k, K$ such that in $J \times J$

$$
k|x-y| \leqq|F(x, z)-F(y, z)| \leqq K|x-y| .
$$

Let $D=R$ or $C$. Let $H: D \times D \times I \times I \rightarrow D$ be continuous, satisfy $\left(\mathrm{H}_{2}\right)$ and

$\left(\mathrm{H}_{3}\right)$ there exists a continuous function $G: D \times D \times I \times I \rightarrow D$ such that

$$
G(u, H(u, v ; x, y) ; x, y) \equiv v .
$$

Let $a, b \in I$ and $a<b$. Then corresponding to any given $f(a)$ and $f(b)$ there is at most one pair of functions $f: I \rightarrow D$ and $g:\{F(x, y): x, y \in I\} \rightarrow D$ such that $|f|$ has a measurable majorant on a subset of $I$ of positive measure and (1) holds.

Proof. Let $f$ and $g$ satisfy the given hypotheses. If $S \subset I$ and $t \in I$ let $F(S, t)=\{F(x, t): x \in S\}$ and $m S$ denote the Lebesgue measure of $S$ (if measurable). There exists a closed and bounded set $E \subset I$ such that $m E>0$ and $f$ is bounded on $E$. Let $I_{1}$ be a closed interval such that $E \subset I_{1} \subset I$. Then $F(E, a) \subset F\left(I_{1}, a\right)$ and $m F(E, a)>0$. Hence there exists a closed interval $I_{0} \subset I_{1}$ such that

$$
m\left\{F(E, a) \cap F\left(I_{0}, a\right)\right\} \geqq \frac{2}{3} m F\left(I_{0}, a\right)>0 .
$$

Let $J$ be a closed interval, $I_{0} \cup[a, b] \subset J \subset I$; then there exists $K>0$ such that (7) holds. Also there exists $S \subset I$ such that $S \supset E, S$ is a finite union of disjoint closed intervals, and

$$
m(S-E) \leqq \frac{1}{\mathbf{6}} K^{-1} m F\left(E \cap I_{0}, a\right) .
$$

If $x \in[a, b]$ then (7) and (9) imply

$$
\begin{aligned}
& m F\left(S \cap I_{0}, x\right)-m F\left(E \cap I_{0}, x\right) \leqq K m\left\{(S-E) \cap I_{0}\right\} \\
& \leqq \frac{1}{6} m F\left(E \cap I_{0}, a\right), \\
& m F\left(E \cap I_{0}, x\right) \geqq m F\left(S \cap I_{0}, x\right)-\frac{\mathbf{1}}{\mathbf{6}} m F\left(E \cap I_{0}, a\right) .
\end{aligned}
$$

But since $F\left(S \cap I_{0}, x\right)$ is a finite sum of closed intervals, $m F\left(S \cap I_{0}, x\right)$ is continuous at $x=a$. Hence there exists $\delta_{1} \in(0, b-a)$ such that if $x \in\left[a, a+\delta_{1}\right]$ then 


$$
\begin{aligned}
m F\left(S \cap I_{0}, x\right) & \geqq m F\left(S \cap I_{0}, a\right)-\frac{1}{6} m F\left(E \cap I_{0}, a\right) \\
& \geqq \frac{5}{6} m F\left(E \cap I_{0}, a\right), \\
m F\left(E \cap I_{0}, x\right) & \geqq \frac{2}{3} m F\left(E \cap I_{0}, a\right) .
\end{aligned}
$$

Similarly there exists $\delta \in\left(0, \delta_{1}\right)$ such that if $x \in[a, a+\delta]$ then $F\left(I_{0}, x\right) \cap F\left(I_{0}, a\right)$ is not empty and

$$
\begin{aligned}
m\left\{F\left(I_{0}, x\right) \cup F\left(I_{0}, a\right)\right\} & <\frac{10}{9} m F\left(I_{0}, a\right), \\
m\left\{F\left(E \cap I_{0}, x\right) \cup F\left(E \cap I_{0}, a\right)\right\} & <\frac{10}{9} m F\left(I_{0}, a\right) .
\end{aligned}
$$

Let $x \in[a, a+\delta]$. If $F\left(E \cap I_{0}, a\right) \cap F\left(E \cap I_{0}, x\right)$ is empty then from (10) and (8)

$$
\begin{aligned}
m\left\{F\left(E \cap I_{0}, a\right) \cup F\left(E \cap I_{0}, x\right)\right\} & =m F\left(E \cap I_{0}, a\right)+m F\left(E \cap I_{0}, x\right) \\
& \geqq \frac{5}{3} m F\left(E \cap I_{0}, a\right) \\
& \geqq \frac{10}{9} m F\left(I_{0}, a\right)
\end{aligned}
$$

which contradicts (11). Hence the intersection is not empty and there exist $s, t \in E \cap I_{0}$ such that $F(s, a)=F(t, x) ;(1)$ and $f(a)=A$ then imply

$$
\begin{aligned}
H(f(s), A ; s, a) & =H(f(t), f(x) ; t, x), \\
f(x) & =G\{f(t), H(f(s), A ; s, a) ; t, x\} .
\end{aligned}
$$

The continuity of $H$ and $G$ imply that $f$ is bounded on $[a, a+\delta]$. Also from (5)

$$
f\left(\alpha_{1}^{k}(x)\right)=G\left(A, h\left\{f\left(\alpha_{1}^{k+1}(x)\right) ; \alpha_{1}^{k+1}(x)\right\} ; a, \alpha_{1}^{k}(x)\right), x \in I, \quad k=0,1, \cdots
$$

Hence $f(x)$ can be expressed as a continuous function of $x$ and $f\left(\alpha_{1}^{n}(x)\right)$, $n=1,2, \cdots$. Since $\alpha_{1}^{n}(b) \rightarrow a$ as $n \rightarrow \infty$, there exists an integer $n$ such that $\alpha_{1}^{n}([a, b]) \subset[a, a+\delta]$. This implies that $f$ is bounded on $[a, b]$ which is sufficient for the proof of the theorem.

Theorem 3. Let $I$ be a convex subset of $R^{n}$. Let $H: D \times D \times I \times I \rightarrow \Delta$ be such that $\left(\mathrm{H}_{1}\right)$ and $\left(\mathrm{H}_{2}\right)$ hold.

Let $\alpha, \beta \in R, \alpha \beta>0$, and $\gamma \in R^{n}$. Let $a_{0}, \cdots, a_{n}$ be the vertices of $a$ non-degenerate simplex in $I$. Then corresponding to any given $f\left(a_{0}\right), \cdots, f\left(a_{n}\right)$, there is at most one pair of functions $f: I \rightarrow D$ and

$$
g:\{\alpha x+\beta y+\gamma: x, y \in I\} \rightarrow \Delta
$$

such that $f$ is locally bounded in $I$ and

$$
g(\alpha x+\beta y+\gamma)=H(f(x), f(y) ; x, y) .
$$

Proof. Let $f$ and $g$ satisfy the given hypotheses. Let $S$ be the set of points in $I$ where $f$ is uniquely determined; then $a_{0}, \cdots, a_{n} \in S$. Let $x, y \in S$ and $x y$ denote the straight line through $x$ and $y$ : 


$$
x y=\{x+s(y-x): s \in R\} .
$$

Then there exists a real interval $\mathscr{I}$ such that

$$
x y \cap I=\{x+s(y-x): s \in \mathscr{I}\} .
$$

Let $f_{1}(s)=f(x+s(y-x)), s \in \mathscr{I}$,

Then

$$
g_{1}(\alpha s+\beta t)=g[(\alpha s+\beta t)(y-x)+\alpha x+\beta x+\gamma], s, t \in \mathscr{I} .
$$

$$
g_{1}(\alpha s+\beta t)=H\left[f_{1}(s), f_{1}(t) ; x+s(y-x), x+t(y-x)\right] .
$$

But $f_{1}$ is locally bounded in $\mathscr{I}, f_{1}(0)=f(x)$ and $f_{1}(1)=f(y)$. By theorem $1 f_{1}$ is uniquely determined in $\mathscr{I}$. Hence $x y \cap I \subset S$ and in particular $S$ is convex. Let $z \in I$ and $M$ be the linear manifold spanned by $S$. Then $M=R^{n}$ and $z \in M$. Hence (see [6] p. 16, Ex. 1.5.1) some straight line through $z$ meets $S$ in a non-degenerate segment, hence in at least two points. Hence $z \in S$ and $I=S$.

\section{References}

[1] J. Aczél, Lectures on Functional Equations and Their Applications, (Academic Press, New York, 1966).

[2] J. Aczél, 'Ein Eindeutigkeitssatz in der Theorie der Funktionalgleichungen und einige ibrer Anwendungen', Acta Math. Acad. Sci. Hung. 15 (1964), 355-362.

[3] J. Aczél, 'Allgemeine Methoden und Eindeutigkeitssatze in der Theorie der Funktionalgleichungen', Jber. Deutsch. Math.-Verein. 67 (1965), 133-142.

[4] J. Aczél and M. Hosszu, 'Further uniqueness theorems for functional equations', Acta Math. Acad. Sci. Hung. 16 (1965), 51-55.

[5] N. Dunford and E. Hille, 'The differentiability and uniqueness of continuous solutions of addition formulas', Bull. Amer. Math. Soc. 53 (1947), $799-805$.

[6] H. G. Eggleston, Convexity (Cambridge University Press, Cambridge, 1958).

[7] T. ח. Howroyd, 'Some Uniqueness Theorems for Functional Equations', J. Australian Math. Soc. 9 (1969), 176-179.

University of Melbourne

Victoria, Australia 